\title{
Effects of the knockdown of death-associated protein 3 expression on cell adhesion, growth and migration in breast cancer cells
}

\author{
UMAR WAZIR $^{1,2}$, ANDREW J. SANDERS ${ }^{3}$, AHMAD M.A. WAZIR ${ }^{4}$, LIN YE $^{3}$, WEN G. JIANG ${ }^{3}$, \\ IRINA C. STER ${ }^{2}$, ANUP K. SHARMA ${ }^{2}$ and KEFAH MOKBEL ${ }^{1,2}$ \\ ${ }^{1}$ The London Breast Institute, Princess Grace Hospital; ${ }^{2}$ Department of Breast Surgery, St. George's Hospital and Medical \\ School, University of London, London; ${ }^{3}$ Cardiff University-Peking University Cancer Institute (CUPUCI), \\ Cardiff University School of Medicine, Cardiff University, Cardiff, Wales, UK; \\ ${ }^{4}$ Peshawar Medical College, Peshawar, Pakistan
}

Received December 30, 2014; Accepted February 4, 2015

DOI: $10.3892 /$ or.2015.3825

\begin{abstract}
The death-associated protein 3 (DAP3) is a highly conserved phosphoprotein involved in the regulation of autophagy. A previous clinical study by our group suggested an association between low DAP3 expression and clinicopathological parameters of human breast cancer. In the present study, we intended to determine the role of DAP3 in cancer cell behaviour in the context of human breast cancer. We developed knockdown sub-lines of MCF7 and MDA-MB-231, and performed growth, adhesion, invasion assays and electric cell-substrate impedance sensing (ECIS) studies of post-wound migration of the cells. In addition, we studied the mRNA expression of caspase 8 and 9, death ligand signal enhancer (DELE), IFN- $\beta$ promoter stimulator 1 (IPS1), cyclin D1 and p21 in the control and knockdown sub-lines. The knockdown sub-lines of MCF7 and MDA-MB-231 had significantly increased adhesion and decreased growth when
\end{abstract}

Correspondence to: Professor Kefah Mokbel, The London Breast Institute, Princess Grace Hospital, 45 Nottingham Place, London W1U 5NY, UK

E-mail:kefahmokbel@hotmail.com

Abbreviations: BAX, Bcl-2-associated X protein; BCL2, B-cell lymphoma 2; BID, BH3 interacting-domain death agonist; cDNA, complementary DNA; DAPs, death-associated proteins; DISC, death-inducing signalling complex; DELE, death ligand signal enhancer; DMEM, Dulbecco's modified Eagle's medium; ECIS, electrical cell impedance sensing; FAK, focal adhesion kinase; FADD, Fas-associated protein with death domain; FCS, foetal calf serum; IPS1, IFN- $\beta$ promoter stimulator 1 ; GAPDH, glyceraldehyde3-phosphate dehydrogenase; LKB1, liver kinase B1; NPI, Nottingham Prognostic Index; qPCR, quantitative polymerase chain reaction; RT, reverse transcription; $\mathrm{tBID}$, truncated $\mathrm{BH} 3$ interacting-domain death agonist; TRAIL, TNF-related apoptosis-inducing ligand

Key words: death-associated protein, DAP3, electrical cell impedance sensing, adhesion, growth assay, invasion, qPCR, breast cancer, apoptosis compared to the controls. Furthermore, invasion and migration were significantly increased in the MDA-MB-231 DAP3kd cells vs. the controls. The expression of caspase 9 and IPS1, known components of the apoptosis pathway, were significantly reduced in the $\mathrm{MCF}^{\text {DAP3kd }}$ cells $(\mathrm{p}=0.05$ and $\mathrm{p}=0.003$, respectively). We conclude that DAP3 silencing contributes to breast carcinogenesis by increasing cell adhesion, migration and invasion. It is possible that this may be due to the activity of focal adhesion kinase further downstream of the anoikis pathway. Further research in this direction would be beneficial in increasing our understanding of the mechanisms underlying human breast cancer.

\section{Introduction}

The role of apoptosis genes in oncogenesis is well attested in the literature, starting with the early study regarding the role of $\mathrm{p} 53$. Since then, a number of other genes have been studied for their role in programmed cell death, including but not limited to B-cell lymphoma 2 (BCL2), Bcl-2-associated $\mathrm{X}$ protein (BAX), and those encoding caspase 3, 6, 8 and 9 . Research on the role of these molecules has greatly improved our understanding of carcinogenesis and have made better prognostication and therapeutic targeting of the underlying pathways possible $(1,2)$.

The death-associated proteins (DAPs) are a relatively novel group of proteins found to have a role in programmed cell death. Four proteins were initially identified: DAP kinase and DAP1, DAP3 and DAP5 (3).

Previously, our group studied DAP3 mRNA expression in archival cancerous and associated normal breast tissue. We found a significant association between low DAP3 expression, higher tumour grade, advanced clinical stage and elevated scoring as per Nottingham Prognostic Index (NPI) (4).

The present literature regarding the locus of DAP3 in cellular pathways suggests at least 3 possible downstream pathways which may be affected by decreased expression of DAP3. One of these functions is mitochondrial protein synthesis, which depends on translocated DAP3 as a major component of mitochondrial ribosomes. This has been characterised as a pro-survival function (5). 
In contrast, DAP3 has also been identified as an important mediator of specific programmes within the external pathway of apoptosis. In association with death ligand signal enhancer (DELE), DAP3 has been characterised as essential for apoptosis initiated by Fas-related death inducing signalling complex (DISC) (6).

In addition, DAP3 has been shown to mediate the interaction of liver kinase B1 (LKB1) with TNF-related apoptosis-inducing ligand (TRAIL)-related DISC. LKB1 has been characterised as a serine-threonine kinase with a tumour-suppressant role in human osteosarcoma cells (7).

Finally, DAP3 plays a role in anoikis by interacting with IFN- $\beta$ promoter stimulator 1 (IPS1) and Fas-associated protein with death domain (FADD). Through IPS1, DAP3 mediates activation of caspase 8 , which represents a critical step in cell detachment-related apoptosis (8). Pertinent to anoikis, caspase 8 cleaves focal adhesion kinase (FAK), which is a critical control element in anoikis signalling, actin cytoskeleton and in cell migration (9).

The results from the previous clinical cohort are consistent with a predominance of the pro-apoptotic function of DAP3 in the context of human breast cancer (4). These findings are consistent with a potential role for DAP3 as a favourable prognostic marker in human breast cancer. Conversely, silencing of DAP3 function appears to foster more aggressive disease, most likely due to corresponding changes in cancer cell behaviour.

Extrapolating from the results from the clinical cohort, one may speculate that silencing of DAP3 expression in breast cancer contributes to increased cell invasiveness, growth, adhesion and migration.

We aimed to study these effects on breast cancer tumour cells after knocking down DAP3 expression in the cells by transfecting them with a plasmid bearing a ribozyme designed for this purpose.

Furthermore, we studied the effects of DAP3 knockdown on the mRNA expression levels of a panel of molecules involved in apoptosis and the cell cycle. The molecules studied as surrogates for apoptosis were DELE, IPS1, caspase 8 and 9. Cyclin D1 and p21 were studied as surrogates for the cell cycle $(10,11)$.

\section{Materials and methods}

Cells and media. The human breast cancer MCF7 and MDA-MB-231 cell lines were purchased from the American Type Culture Collection (ATCC; Rockville, MD, USA) and routinely maintained in Dulbecco's modified Eagle's medium (DMEM), supplemented with $10 \%$ foetal calf serum (FCS) and antibiotics. The cells were incubated at $37^{\circ} \mathrm{C}$ in $5 \% \mathrm{CO}_{2}$ and $95 \%$ humidity.

Generation of MCF-7 and MDA-MB-231 cells displaying knockdown of DAP3. DAP3 expression was suppressed in the MCF7 and MDA-MB-231 cells through transfection of a pEF6/V5-His-TOPO (Invitrogen, Paisley, UK) containing ribozyme transgenes which specifically recognised and cleaved the DAP3 mRNA transcript. These techniques have been widely implemented by our laboratories and are outlined by Jiang et al (12). The purified plasmids were sequenced in order to verify the presence and sequence of the construct
(MWG, Milton Keynes, UK). MCF7 and MDA-MB-231 cells were transfected with plasmids containing the ribozyme transgenes or an empty pEF6 control plasmid. Cells were then subjected to selection with blasticidin. The cell lines containing the control pEF6 plasmid were designated as $\mathrm{MCF} 7^{\mathrm{PEF} 6}$ and MDA-MB-231 ${ }^{\mathrm{pEF} 6}$ respectively, and those containing the ribozyme transgenes were labelled MCF7 ${ }^{\text {DAP3kd }}$ and MDA-MB-231 ${ }^{\text {DAP3kd }}$, respectively.

The plasmids were previously produced and provided by Jia et al (13). Knockdown of DAP3 was demonstrated in transfected mammalian cells using conventional and real-time quantitative polymerase chain reaction (qPCR) (Fig. 1).

Quantitative analysis of transcripts in the cells. Total RNA was extracted from cell pellets using Triagent obtained from Sigma-Aldrich. RNA was extracted and purified from the cells using a modified phenol-chloroform phase separation technique as per the manufacturer's instructions. Equal amounts of RNA were reverse transcribed into complementary DNA (cDNA) using a first-strand reverse transcription (RT) kit from AbGene (Surrey, UK). For quantitative analysis of the DAP3 transcripts, we employed a real-time quantitative PCR assay, as previously reported (14). Primers (sequences shown in Table I) were designed using the Beacon Designer software. The Ampliflor Uniprimer system was used as the probe system.

cDNA from cells and tissues, along with a set of standards were amplified simultaneously on an iCycler iQ5 system (Bio-Rad). The concentration of the respective transcript was calculated from the standard curve which was simultaneously generated. The levels of the transcripts are shown here as the respective transcript/glyceraldehyde3-phosphate dehydrogenase (GAPDH) ratio.

Growth assay for the breast cancer cell lines. The growth of the breast cancer cell lines was assessed using a colorimetricbased method (15). On days 1 (D1), 3 (D3) and 5 (D5), the plates were fixed and stained with crystal violet. Cells (3,000/well) were added. Cell density and growth were measured on a Bio-Tek ELx800 multiplate reader (Bio-Tek Instruments, Inc., Winooski, VT, USA). During each run, each cell line was tested in eight repeats, of whom an average was taken. The percentage of increase over the readings taken on D1 was calculated for D3 and D5.

Adhesion assay for the breast cancer cell lines. This was based on a previously published protocol (14). In brief, Matrigel basement membrane matrix purchased from BD Biosciences (Oxford, UK), was used as an adhesion sheet at $5 \mu \mathrm{g} /$ well. Cells $(45,000 /$ well $)$ were added. After a 40-min incubation, the wells were vigorously washed to remove unbound cells. Adherent cells were then fixed and stained with crystal violet. Stained cells were later counted in a number of random fields under a x40 objective.

Invasion assay for the breast cancer cell lines. Invasion assays were undertaken using inserts with $8-\mu \mathrm{m}$ pores from BD Biosciences, in 24-well tissue culture plates. Each insert was first coated with Matrigel ${ }^{\mathrm{TM}}$. After rehydration, 15,000 cells were seeded into each insert. After 3 days of incubation, the inserts were fixed with formalin and stained 
Table I. Primers used in the polymerase chain reaction.

\begin{tabular}{|c|c|}
\hline Gene & Sequence $\left(5^{\prime}-3^{\prime}\right)$ \\
\hline PDPL F8 & GAATCATCGTTGTGGTTATG \\
\hline PDPL ZR1 & ACTGAACCTGACCGTACACTTTCATTTGCCTATCACAT \\
\hline DAP1 F1 & ATGGACAAGCATCCTTCC \\
\hline DAP1 ZR1 & ACTGAACCTGACCGTACACTCTGTCAGGGAAATACCAA \\
\hline DAP3 F1 & AAAGCACTGAGAAAGGGAGT \\
\hline DAP3 ZR1 & ACTGAACCTGACCGTACACCTCTTTAGCTCTTTCAGCA \\
\hline Caspase 8 F1 & AGAAAGGAGGAGATGGAAAG \\
\hline Caspase 8 ZR1 & ACTGAACCTGACCGTACAGACCTCAATTCTGATCTGCT \\
\hline Caspase 9 F1 & AAGCCCAAGCTCTTTTTC \\
\hline Caspase 9 ZR1 & ACTGAACCTGACCGTACAGTTACTGCCAGGGGACTC \\
\hline Caspase 9 F2 & ATATGATCGAGGACATCCAG \\
\hline Caspase 9 ZR2 & ACTGAACCTGACCGTACACAGGAGATGAACAAAGGAAG \\
\hline DELE F1 & GTCATGAGCATGGCAGAG \\
\hline DELE ZR1 & ACTGAACCTGACCGTACAACCTGGCATAGCGCTACT \\
\hline IPS1 F1 & AGAGAAGGAGCCAAGTTACC \\
\hline IPS1 ZR1 & ACTGAACCTGACCGTACAATTCCTTGGGATGGCTCT \\
\hline P21 F1 & CGGGATGAGTTGGGAGGAG \\
\hline P21 ZR1 & ACTGAACCTGACCGTACAACAGGTCCACATGGTCTTCC \\
\hline Cyclin D1 F1 & CGGTGTCCTSCTTCAAATGT \\
\hline Cyclin D1 ZR1 & ACTGAACCTGACCGTACAAAGCGGTCCAGGTAGTTC \\
\hline GAPDH F1 & AAGGTCATCCATGACAACTT \\
\hline GAPDH ZR1 & ACTGAACCTGACCGTACAGCCATCCACAGTCTTCTG \\
\hline GAPDH F2 & CTGAGTACGTCGTGGAGTC \\
\hline GAPDH ZR2 & ACTGAACCTGACCGTACACAGAGATGATGACCCTTTTG \\
\hline
\end{tabular}

PDPL, podoplanin; DAP1, death-associated protein 1; DELE, death ligand signal enhancer; IPS1, IFN- $\beta$ promoter stimulator-1; p21, protein 21; GAPDH, glyceraldehyde-3-phosphate dehydrogenase.

in crystal violet. Cells stained with crystal violet were counted and observed under a microscope for possible invasion as described by Jiang et al (16).

Electric cell-substrate impedance sensing (ECIS)-based cell adhesion and cell migration assays. ECIS Z $\theta$ device and 96W1E arrays were used (Applied Biophysics Inc., Troy, NY, USA) (17). Prior to seeding, the wells were instilled with serum-free medium, and the array was subjected to the ECIS Z $\theta$ machine's 'stabilisation' function. A plate plan was made after thus identifying non-functional wells.

An identical number of MCF7 or MDA-MB-231 cells of the control or knockdown sub-lines were added into each well. Cell adhesion was recorded immediately following addition of the cells at multiple frequencies, for up to $3 \mathrm{~h}$. For the cell migration assay, confluent cells were wounded after at least $10 \mathrm{~h}$ of growth. Wounding conditions were optimised for each cell line (for MDA-MB-231, $20 \mathrm{sec}, 3,000 \mu \mathrm{A}, 60,000 \mathrm{~Hz}$; for MCF7, $20 \mathrm{sec}, 1,400 \mu \mathrm{A}, 60,000 \mathrm{~Hz}$ ). The migration of the cells was immediately traced following wounding for up to $4 \mathrm{~h}$, again with multiple frequencies. The knockdown sub-lines were compared to the controls. Cell migration is shown here as the change in resistance. The migration over the 4-h post wounding in the control and control cell lines was compared using a random effects model, in which an average curve was calculated and then were compared for significant differences.
Statistical analysis. Analysis of the data was performed using the SigmaPlot 11 statistical software package (Systat Software, San Jose, CA, USA). Medians were compared using the Mann-Whitney U test, while means were compared using the two-sample t-test. Percentage control was employed to compensate for variations between repeats of assays likely due to passage, variations in quality of material and performance of technique.

After consultation with the university statistician, it was found to be appropriate to apply random effects modelling to the results of a representative instance of the ECIS-based migration assay in order to ascertain the significance of the results.

\section{Results}

Decreased growth is observed in the knockdown sub-lines compared to the controls. The experiment was repeated successfully 4 times $(n=4)$. Triplicate plates were made for D1 (reference plate), D3 and D5.

Compared to the controls $\left(\mathrm{MCF}^{\mathrm{pEF} 6}\right)$, the DAP3 knockdown strain (MCF7 ${ }^{\text {DAP3kd }}$ ) was found to show less growth on D3 (100 vs. 55.7; $\mathrm{p}=0.029)$ and on D5 (100 vs. 62.5; $\mathrm{p}=0.029)$. A similar trend was observed in the MDA-MB-231 sub-lines, which achieved statistical significance on D5 (100 vs. 69.7; $\mathrm{p}=0.01$ ) (Fig. 2). 
A

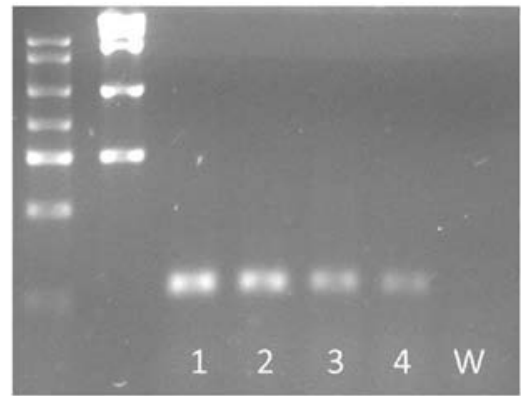

\begin{tabular}{|c|c|c|c|c|c|}
\hline Legend & Sub-line & Area & Mean & Min & Max \\
\hline 1 & MCF7PEF6 & & & & \\
\hline 2 & MCF7DAP3kd & 0.013 & 3548.98 & 2736 & 4095 \\
\hline 3 & MDA-MB-231pEF6 & 0.013 & 3440.897 & 2800 & 4014 \\
\hline 4 & MDA-MB-231DAP3kd & 0.013 & 3136.149 & 2752 & 3544 \\
\hline & & 0.013 & 2906.394 & 2655 & 3237 \\
\hline W & Water & 0.013 & 2576.52 & 2444 & 2865 \\
\hline
\end{tabular}

B

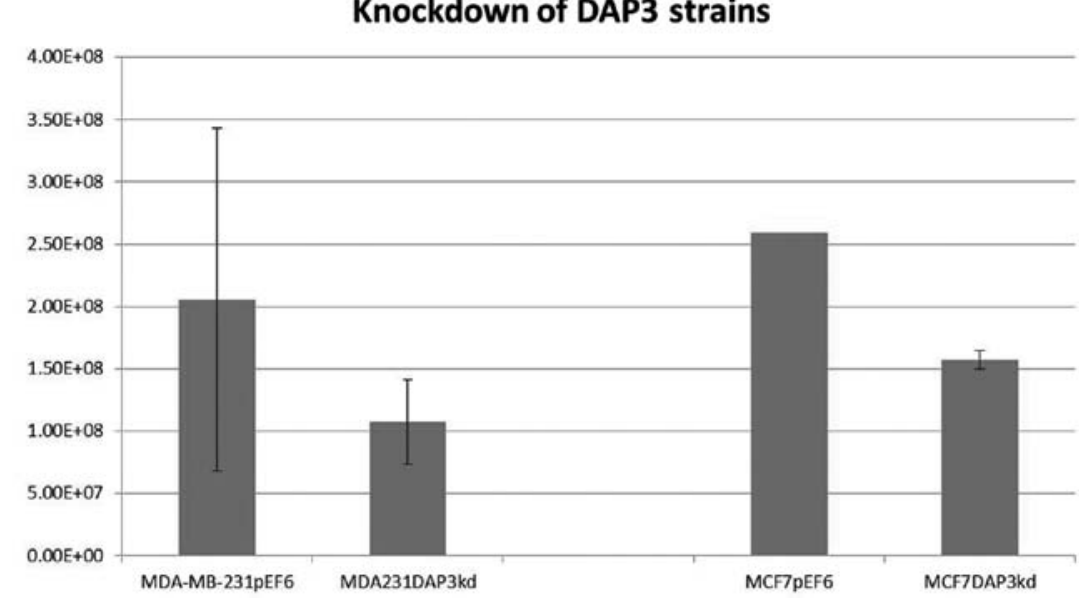

Figure 1. Demonstration of the knockdown of DAP3 mRNA expression in the MCF7 and MDA-MB-231 sub-lines using conventional and quantitative PCR. (A) PCR gel with densitometry by ImageJ; (B) knockdown demonstrated by quantitative real-time PCR.

5 Day Growth assay in MCF7 and MDA-MB-231 control and DAP3 knockdown cell lines
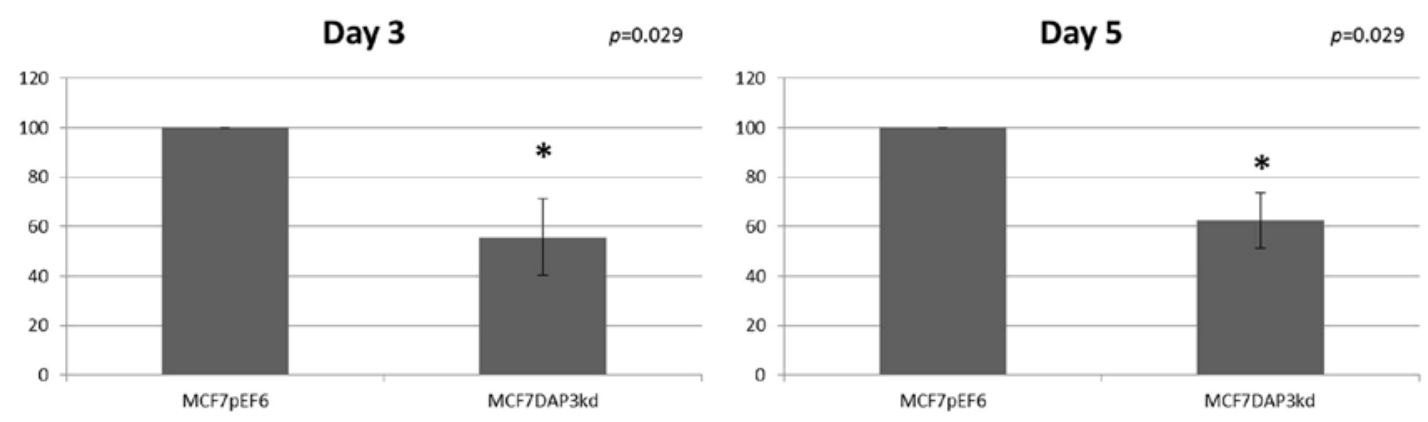

Day 3
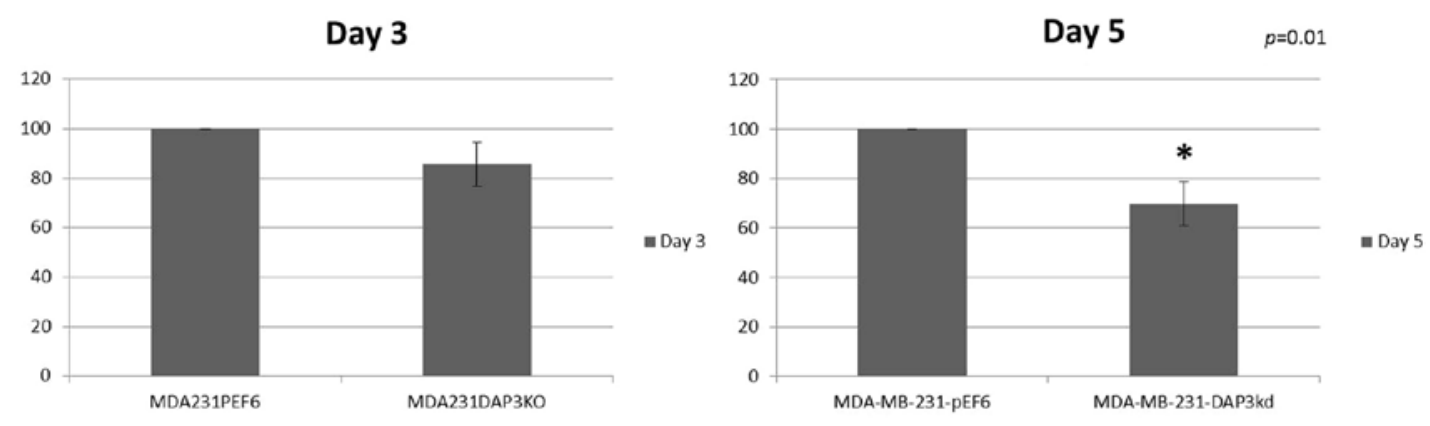

*significant

Figure 2. Growth assay comparing the controls (pEF6) and DAP3 knockdown sub-lines of MCF7 and MDA-MB-231. The results of a 5-day growth assay, with plates fixed on day 1 (D1; reference), day 3 (D3) and day 5 (D5). Each cell line had 5 repeats (n=4) ("significant associations, Mann-Whitney U test).

Increased adhesion observed in the knockdown sub-lines compared to the controls. The adhesion assay was performed at least 3 times ( $\mathrm{n}=4$ for MCF7; $\mathrm{n}=3$ for MDA-MB-231), with eight repeats for each sub-line studied. 
Adhesion assay in control and DAP3 knockdown cell lines
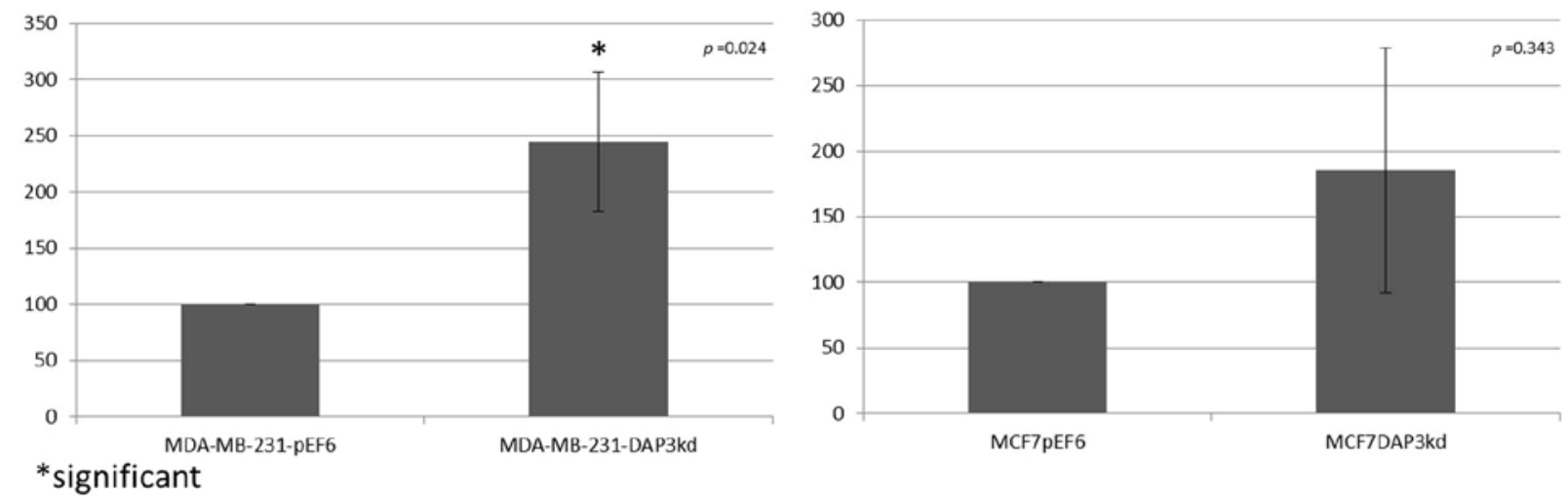

Figure 3. Adhesion assay comparing the controls (pEF6) and DAP3 knockdown sub-lines of MCF7 and MDA-MB-231. The assay was performed on Matrigelcoated wells, with 6 repeats/cell line. The plate was incubated for $45 \mathrm{~min}$ before fixation in $4 \%$ formalin $(\mathrm{n}=4)$ ("significant associations, Mann-Whitney $\mathrm{U}$ test).

\section{Invasion assay in control and DAP3 knockdown cell lines}
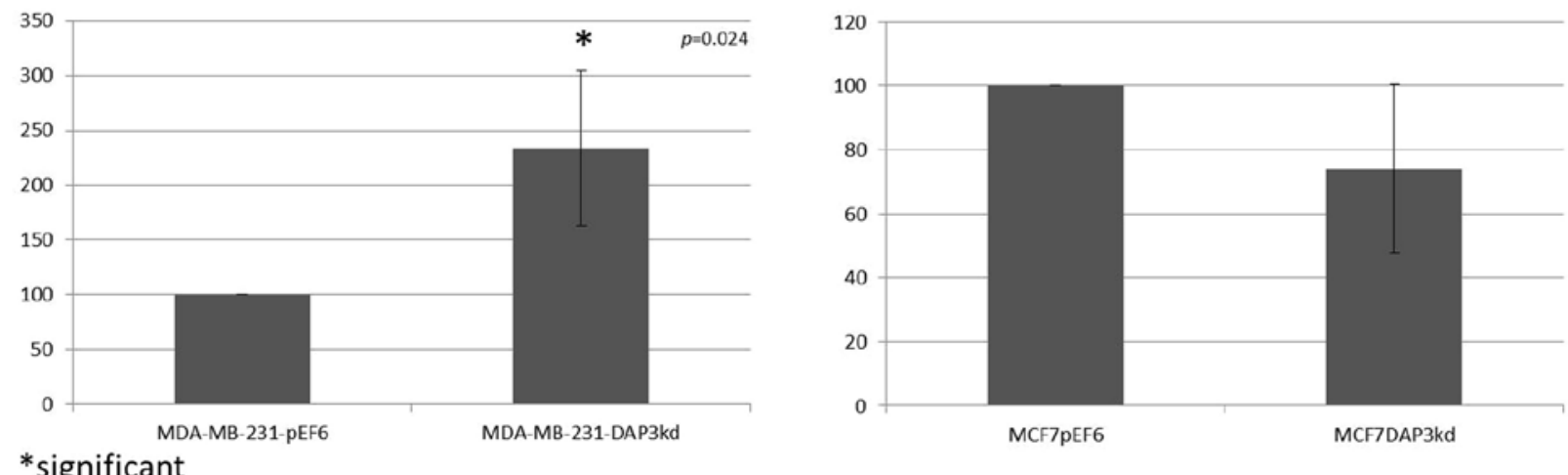

*significant

Figure 4. Invasion assay comparing the controls (pEF6) and DAP3 knockdown sub-lines of MCF7 and MDA-MB-231. The assay was performed on Matrigel-coated inserts suspended in nutrient medium. The plate was incubated until day 3 before fixation in $4 \%$ formalin $(\mathrm{n}=4)$ ("significant associations, Mann-Whitney $\mathrm{U}$ test).

In the MCF7 cell lines, DAP3 knockdown resulted in a consistently higher ability to adhere as compared to the controls. However, this trend did not achieve statistical significance $(\mathrm{p}=0.34)$.

As was observed in the MCF7 sub-lines, MDA-MB$231^{\text {DAP3kd }}$ showed a consistent trend promoting adhesion when compared to MDA-MB-231 $1^{\mathrm{EFF}}$. This trend was much more pronounced, and was found to be statistically significant (245 vs. $100 \%$; $\mathrm{p}=0.024$ ) (Fig. 3).

Increased invasion observed in the MDA-MB-231 DAP3kd cells compared to the controls. The Matrige ${ }^{\mathrm{TM}}$ based invasion was performed repeatedly in both cell lines $(n=3)$. There was a significant increase in invasion noted in the MDA-MB$231^{\text {DAP3kd }}$ cells vs. the controls $(\mathrm{p}=0.024)$. However, no clear change in invasive ability was noted in the knockdown strains when compared to the controls in MCF7 (Fig. 4).

Reduction of expression of pro-apoptotic molecules in the $M C F 7^{D A P 3 k d}$ and MDA-MB-231 DAPSkd cells. After knockdown was confirmed, the mRNA expression levels of the following molecules were studied in the cDNA libraries derived from the transfected MCF7 and MDA-MB-231 sub-lines: caspase 8, caspase 9, IPS1, DELE, p21 and cyclin D1.
$\mathrm{MCF}^{\mathrm{DAP} 3 k \mathrm{~d}}$ showed a marginally significant depression in the mRNA expression of caspase 9 (MCF $7^{\mathrm{pEF}}{ }^{\mathrm{V}} \mathrm{vs}$. MCF7 ${ }^{\mathrm{DAP} 3 k \mathrm{~d}}$ : 100 vs. $63.5 ; \mathrm{p}=0.05)$, which is an apical caspase associated with the internal apoptosis pathway (Fig. 5).

Furthermore, the expression of IPS1 was also suppressed in MCF7 ${ }^{\text {DAP3kd }}$ vs. the controls (MCF7 ${ }^{\text {EFF6 }}$ vs. MCF7 ${ }^{\text {DAP3kd: }} 100$ vs. 14.5; $\mathrm{p}=0.003$ ) (Fig. 5).

No statistically significant difference was noted in the expression of caspase 8, cyclin D1, DELE and p21 (data not shown).

In vitro migration assay using ECIS. When compared to the control (MCF7 $7^{\mathrm{pEF} 6}$ ), the DAP3 knockdown sub-line demonstrated consistently greater attachment and migration across at least 3 repeats. Presented here are the results of a representative instance of the ECIS assay (Fig. 6). The curve for MCF $7^{\mathrm{pEF} 6}$ was calculated as follows:

$$
\text { Readings }=-2.69-6.22 \times \text { time }+13.72 \times \text { time }^{2}
$$

In the case of MCF7 ${ }^{\text {DAP3kd }}$, the equation was calculated as follows:

$$
\text { Readings }=-20.69-27.38-6.22 \times \text { time }+13.72 \times \text { time }^{2}
$$

The difference in the readings in the 2 groups failed to achieve statistical significance $(p=0.27)$. MDA-MB-231 


\section{Caspase 9}
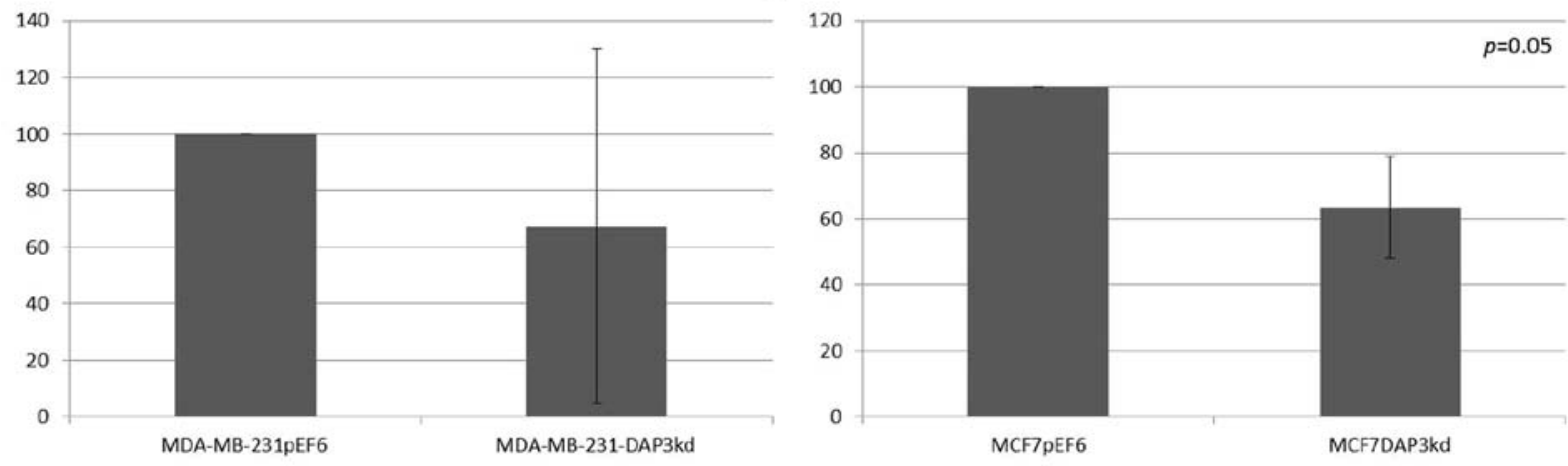

IPS1
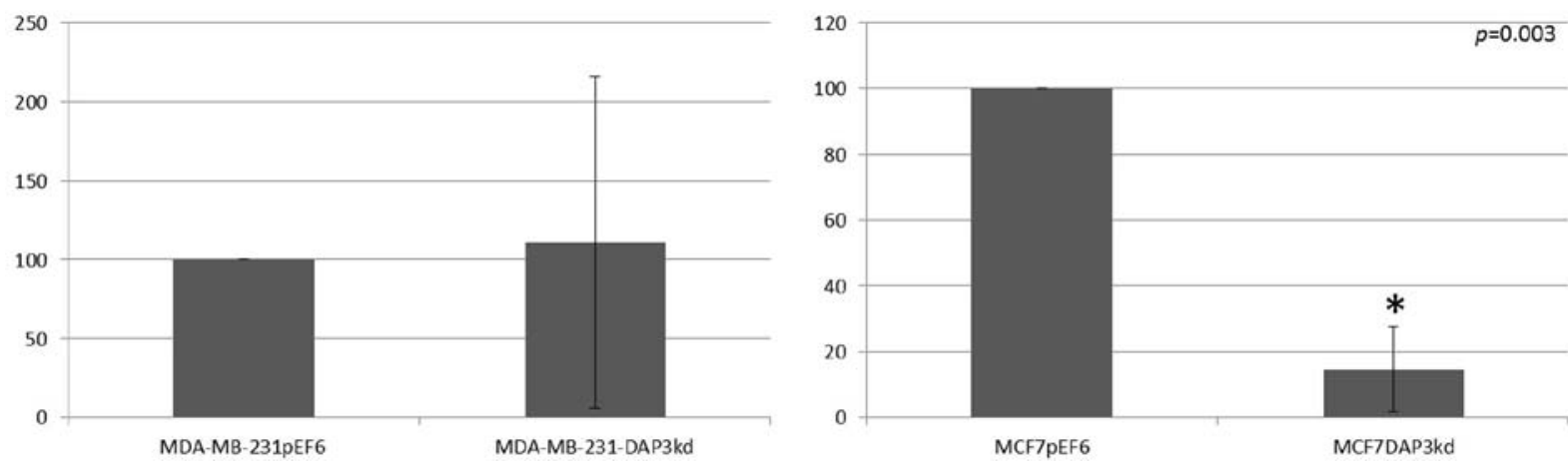

Figure 5. mRNA expression of IPS1 (IFN- $\beta$ promoter stimulator-1) and caspase 9 in the control and DAP3 knockdown sub-lines ("significant associations, Mann-Whitney U test).

sub-lines were similarly studied. When compared to controls (MDA-MB-231 ${ }^{\text {pEF6}}$ ), MDA-MB-231 ${ }^{\text {DAP3kd }}$ knockdown sub-line demonstrated consistently greater attachment and migration across at least 3 repeats. These effects were much more pronounced in comparison to those noted in the MCF7 sublines (Fig. 6). The curve for MDA-MB-231 ${ }^{\mathrm{pEF} 6}$ was calculated as follows:

$$
\text { Readings }=-19.56-9.70 \times \text { time }+4.58 \times \text { time }^{2}
$$

The curve for MDA-MB-231 ${ }^{\text {DAP3kd }}$ was calculated as follows:

Readings $=-19.56-63.68+(-9.70+218.9087) \times$ time $+4.58 \times$ time $^{2}$

There was strong evidence that the growth readings in the 2 groups differed substantially $(\mathrm{p}<0.001)$.

\section{Discussion}

DAP3 has long been known to be a component of the external apoptosis pathway, and to be critical for certain programmes within the apoptosis pathway. While it would be reasonable to characterise DAP3 as a potentially tumour-suppressant molecule on the basis of its pro-apoptotic function, there was no prior accessible literature which would support such a hypothesis. In view of the co-existing mitochondrial role of DAP3, such a hypothesis was at best speculative without further evidence $(5,8)$.

In the course of a previous study of DAP3 mRNA expression in a clinical cohort of archival breast cancer tissue, we found significant inverse associations between DAP3 expression and tumour grade, clinical stage and clinical outcome. This was in keeping with our hypothesis regarding the role of DAP3 as a tumour-suppressant gene due to its pro-apoptotic function (4).

The results we observed in the present in vitro assays help further clarify the role that DAP3 may potentially have in human breast cancer. Significant increases in adhesion were noted in the knockdown sub-lines as compared to the controls, while increased invasion and migration were observed in MDA-MB-231 ${ }^{\text {DAP3kd }}$ vs. MDA-MB-231 ${ }^{\mathrm{pEF} 6}$. These findings are in keeping with the inference one may draw from the clinical study that DAP3 silencing could predispose the emergence of more aggressive disease. Furthermore, the implication of these observations is that DAP3 knockdown may contribute to more aggressive disease by increasing the ability of cells to migrate from the primary tumour, and to adhere and invade secondary sites. As one may recall from the discussion pertaining to the downstream pathway of DAP3, the most likely effector of this could be FAK, which would be cleaved by caspases in the presence of active DAP3. FAK is known to play an important role in cell adhesion and motility and in the regulation of anoikis (9).

In contrast, the suppression of growth in the knockdown sub-lines compared to the controls cannot be characterised as evidence of an antioncogenic function of DAP3 in breast cancer. However, it is in keeping of the pro-survival role of DAP3 in mitochondrial protein synthesis, and reiterates the centrality of the locus of DAP3 in cellular metabolism.

Furthermore, the suppression of IPS1 expression observed in the MCF7 ${ }^{\text {DAP3kd }}$ cells compared to the controls suggests an increased involvement of the anoikis programmed within 

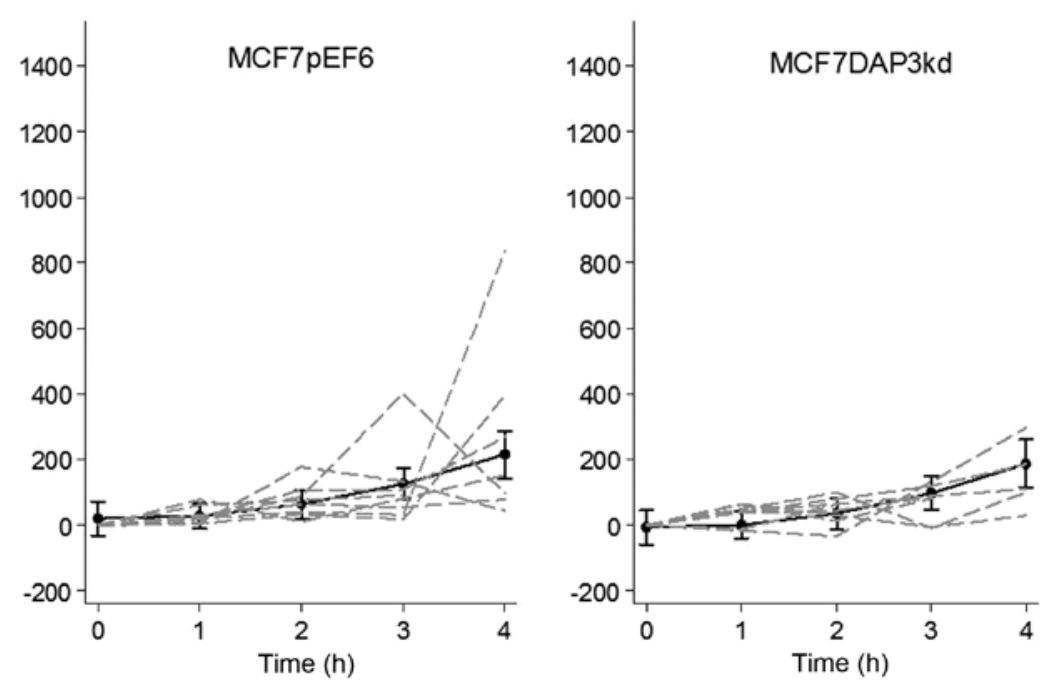

$\rightarrow$ Predicted means

$\longmapsto 95 \% \mathrm{Cl} \quad--$ observed curves

MCFF $^{\text {pEF6 }}$ vs. MCF7 ${ }^{\text {DAP3kd: }} p=0.24$
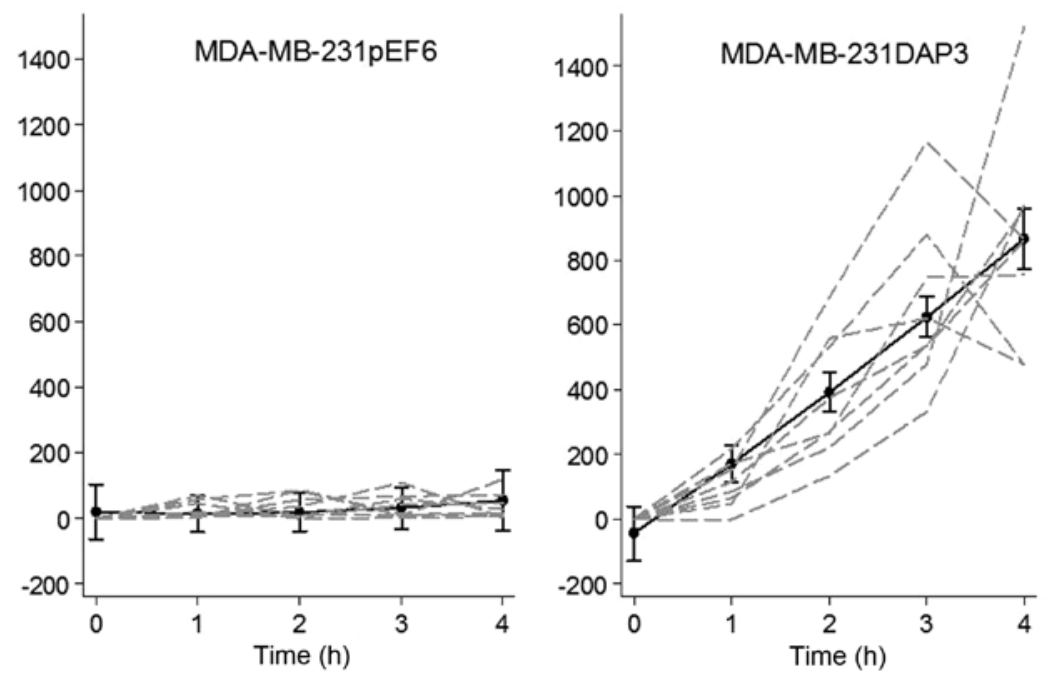

$\rightarrow$ Predicted means

$\longmapsto 195 \% \mathrm{Cl} \quad--$ observed curves

MDA-MB-231 ${ }^{\text {pEF6 }}$ vs. MDA-MB-231 ${ }^{\text {DAP1kd: }} p=0.001$

Figure 6. ECIS-based post-wounding migration assay of DAP3 knockdown and control sub-lines. $\mathrm{x}$-axis represents the change in impedance after successful wounding of the cell monolayer ("significant associations). DAP3, death-associated protein 3; ECIS, electric cell-substrate impedance sensing.

the apoptosis pathway in breast carcinogenesis, which in turn supports our previously posited hypothesis of an involvement of FAK in the increase noted in cellular migration, adhesion and invasion (18).

Finally, the marginally significant suppression of caspase 9 expression observed in MCF7 ${ }^{\text {DAP3kd }}$ is consistent with the activation of pro-caspase 9 as a result of MOMP due to the action of truncated $\mathrm{BH} 3$ interacting-domain death agonist (tBID), which in turn is the result of the action of caspase 8 on BID. The lack of a significant effect on caspase 8 expression may be ascribed in part to the multiplicity of inputs received by this pathway other than those from DAP3, and in part to the limited number of repeats in the assay (19).
Conclusion and future directions. In the course of the present study, the DAP3 knockdown MCF7 sub-line shown a reduced response to serum starvation compared to the controls in an apoptosis assay using Annexin V/propidium staining and flow cytometry. However, due to limitations in time and disruptions due to relocation of the laboratory, we were unable to reproduce the assay with comparable parameters and conditions.

Future apoptosis studies on DAP3 knockdown breast cancer cell sub-lines incubated in various death receptor ligands would be useful to delineate the specific DISCs pertinent to DAP3 function in the context of breast cancer. Furthermore, the changes in the protein expression levels of known and suspected downstream effectors in DAP3 knock- 
down cells would be instrumental for better characterisation of the pathways most likely to account for the in vitro assays. Of particular interest would be the expression of FAK, which may potentially account for the effects noted in the adhesion and invasion assays.

Furthermore, studies into mitochondrial function in such cells would be pertinent, particularly with regards to the findings of the growth assay.

In summary, our results are highly suggestive of a significant role for DAP3 as a tumour-suppressant gene in the context of human breast cancer, and that a decrease in DAP3 expression may be associated with an increase in cancer cell invasiveness, migration and adhesion, which would be prerequisites of more aggressive disease. The apparently anomalous finding regarding cell growth in the MCF7 cells reiterates the potentially contradictory locus of DAP3 in critical cellular pathways, which highlights the potential importance of DAP3 in the coordination of these disparate processes. A more nuanced understanding of the pathways at play would go a long way in furthering our understanding of breast carcinogenesis, and could potentially improve prognostication and increase the options available in terms of the therapeutic targeting of human breast cancer.

\section{Acknowledgements}

The present study was funded by grants from the Breast Cancer Hope Foundation (London, UK).

\section{References}

1. Elmore S: Apoptosis: A review of programmed cell death. Toxicol Pathol 35: 495-516, 2007.

2. Rastogi RP, Sinha R and Sinha RP: Apoptosis: Molecular mechanisms and pathogenicity. EXCLI J 8: 155-181, 2009.

3. Levy-Strumpf N and Kimchi A: Death associated proteins (DAPs): From gene identification to the analysis of their apoptotic and tumor suppressive functions. Oncogene 17: 3331-3340, 1998

4. Wazir U, Jiang WG, Sharma AK and Mokbel K: The mRNA expression of DAP3 in human breast cancer: Correlation with clinicopathological parameters. Anticancer Res 32: 671-674, 2012.

5. Kim HR, Chae HJ, Thomas M, Miyazaki T, Monosov A, Monosov E, Krajewska M, Krajewski S and Reed JC: Mammalian dap3 is an essential gene required for mitochondrial homeostasis in vivo and contributing to the extrinsic pathway for apoptosis. FASEB J 21: 188-196, 2007.
6. Harada T, Iwai A and Miyazaki T: Identification of DELE, a novel DAP3-binding protein which is crucial for death receptormediated apoptosis induction. Apoptosis 15: 1247-1255, 2010.

7. Takeda S, Iwai A, Nakashima M, et al: LKB1 is crucial for TRAIL-mediated apoptosis induction in osteosarcoma. Anticancer Res 27: 761-768, 2007.

8. Li HM, Fujikura D, Harada T, Uehara J, Kawai T, Akira S, Reed JC, Iwai A and Miyazaki T: IPS-1 is crucial for DAP3mediated anoikis induction by caspase- 8 activation. Cell Death Differ 16: 1615-1621, 2009.

9. Lauricella M, Ciraolo A, Carlisi D, Vento R and Tesoriere G: SAHA/TRAIL combination induces detachment and anoikis of MDA-MB231 and MCF-7 breast cancer cells. Biochimie 94: 287-299, 2012.

10. Tobin NP, Sims AH, Lundgren KL, Lehn S and Landberg G: Cyclin D1, Id1 and EMT in breast cancer. BMC Cancer 11: 417 , 2011.

11. Liu Y, Encinas M, Comella JX, Aldea M and Gallego C: Basic helix-loop-helix proteins bind to $\operatorname{TrkB}$ and $p 21^{\text {Cipl }}$ promoters linking differentiation and cell cycle arrest in neuroblastoma cells. Mol Cell Biol 24: 2662-2672, 2004.

12. Jiang WG, Grimshaw D, Lane J, Martin TA, Abounader R, Laterra $\mathbf{J}$ and Mansel RE: A hammerhead ribozyme suppresses expression of hepatocyte growth factor/scatter factor receptor c-MET and reduces migration and invasiveness of breast cancer cells. Clin Cancer Res 7: 2555-2562, 2001.

13. Jia Y, Ye L, Ji K, Toms AM, Davies ML, Ruge F, Ji J, Hargest R and Jiang WG: Death associated protein 1 is correlated with the clinical outcome of patients with colorectal cancer and has a role in the regulation of cell death. Oncol Rep 31: 175-182, 2014.

14. Sanders AJ, Parr C, Mason MD and Jiang WG: Suppression of hepatocyte growth factor activator inhibitor-1 leads to a more aggressive phenotype of prostate cancer cells in vitro. Int J Mol Med 20: 613-619, 2007.

15. Jiang WG, Redfern A, Bryce RP and Mansel RE: Peroxisome proliferator activated receptor-gamma (PPAR-gamma) mediates the action of gamma linolenic acid in breast cancer cells. Prostaglandins Leukot Essent Fatty Acids 62: 119-127, 2000.

16. Jiang WG, Martin TA, Lewis-Russell JM, Douglas-Jones A, Ye L and Mansel RE: Eplin-alpha expression in human breast cancer, the impact on cellular migration and clinical outcome. Mol Cancer 7: 71, 2008.

17. Keese CR, Wegener J, Walker SR and Giaever I: Electrical wound-healing assay for cells in vitro. Proc Natl Acad Sci USA 101: 1554-1559, 2004.

18. Sood AK, Coffin JE, Schneider GB, Fletcher MS, DeYoung BR, Gruman LM, Gershenson DM, Schaller MD and Hendrix MJ: Biological significance of focal adhesion kinase in ovarian cancer: Role in migration and invasion. Am J Pathol 165: 1087-1095, 2004

19. Valentijn AJ and Gilmore AP: Translocation of full-length Bid to mitochondria during anoikis. J Biol Chem 279: 32848-32857, 2004. 